\title{
Composite panels for optical mirrors for Cherenkov telescopes: development of the cold glass slumping technology
}

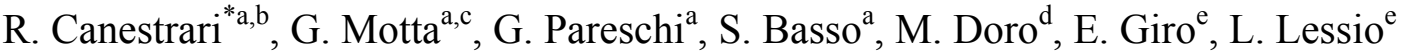 \\ ${ }^{a}$ INAF-Osservatorio Astronomico di Brera - Via Bianchi, 4623807 Merate (Lc) Italy \\ ${ }^{\mathrm{b}}$ Università degli Studi dell'Insubria - Via Valleggio, 1122100 Como (Co) Italy \\ c Università degli Studi di Milano-Bicocca - Piazzale dell’Ateneo Nuovo, 120126 Milano Italy \\ ${ }^{\mathrm{d}}$ INFN-Padova - Via Marzolo 835131 Padova \\ ${ }^{\mathrm{e}}$ INAF-Osservatorio Astronomico di Padova - Vicolo Osservatorio 535122 Padova Italy
}

\begin{abstract}
In the last decade a new window for ground-based high energy astrophysics has been opened. It explores the energy band from about $100 \mathrm{GeV}$ to $10 \mathrm{TeV}$ making use of Imaging Atmospheric Cherenkov Telescopes (IACTs). Research in Very High Energy (VHE) gamma-ray astronomy is improving rapidly and thanks to the newest facilities as MAGIC, HESS and VERITAS astronomers and particle physicists are obtaining surprising implications in the theoretical models.

New projects have been started as the European Cherenkov Telescope Array (CTA) and the U.S. Advanced Gamma-ray Imaging System (AGIS). The aim is to enhance both the sensitivity and the energy band coverage to perform imaging, photometry and spectroscopy of sources. In this framework, tens of thousands of optical mirror panels have to be manufactured, tested and mounted into the telescopes. Because of this high number of mirrors it is mandatory to develop a technique easily transferable to industrial mass production, but keeping the technical and cost-effectiveness requirements of the next generation of $\mathrm{TeV}$ telescopes.

In this context the Astronomical Observatory of Brera (INAF-OAB) is investigating a technique for the manufacturing of stiff and lightweight glass mirror panels with modest angular resolution. These panels have a composite sandwich-like structure with two thin glass skins on both sides of a core material; the reflecting skin is optically shaped using an ad-hoc slumping procedure. The technology here presented is particularly attractive for the mass production of cost-effective mirror segments with long radius of curvature like those required in the primary mirrors of the next generation of Cherenkov telescopes. In this paper we present and discuss some relevant results we have obtained from the latest panels realized.
\end{abstract}

Keywords: Segmented Optics, Glass Slumping, lightweight optics, Cherenkov telescopes, CTA, AGIS

\section{INTRODUCTION}

Gamma rays at $\mathrm{MeV}-\mathrm{GeV}$ energies have been typically observed with space-based instruments but at higher energies those instruments are completely unusable due to the very low flux: few photons per $\mathrm{m}^{2}$ per year above $1 \mathrm{TeV}$ for strong sources. With the advent of the ground-based IACTs in late 1980's, the observations of VHE gamma-rays came into reality and since the discovery of the TeV emission from the Crab Nebula by Whipple in 1989 [1] this astronomy has achieved exceptional results. At the moment of writing, thanks to the high sensitivity of the present days Cherenkov telescopes, there are more then 100 sources with a detected TeV emission (see Fig. 1). Both galactic and extragalactic sources have been detected using instruments located on the Southern and Northern hemispheres. Those instruments can reach energy threshold below $70 \mathrm{GeV}$ by using very large collectors such as for the two $17 \mathrm{~m}$ MAGIC telescopes sited in

*rodolfo.canestrari@brera.inaf.it

Modern Technologies in Space- and Ground-based Telescopes and Instrumentation, edited by Eli Atad-Ettedgui, Dietrich Lemke, Proc. of SPIE Vol. 7739, 77390H - @ 2010 SPIE · CCC code: 0277-786X/10/\$18 - doi: 10.1117/12.857268 
La Palma (Canary Islands). Or they can have an enhanced angular resolution thanks to the high performances achievable with stereoscopy in telescope arrays such as H.E.S.S. in Namibia or VERITAS in Arizona (USA).
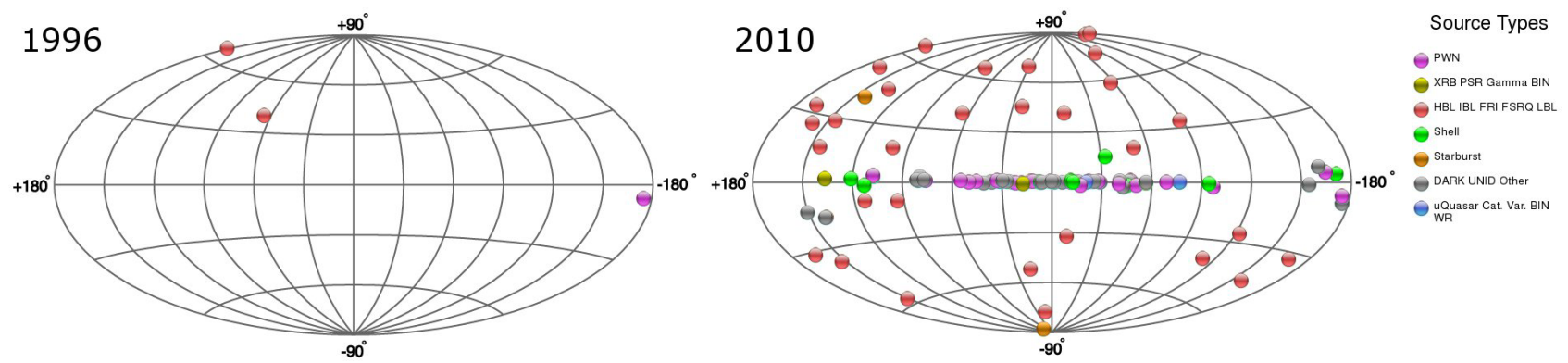

Fig. 1. Sky maps showing the improvement in number of sources detected in the VHE astrophysics from 1996 (left panel) to 2010 (right panel). (Images created using http://tevcat.uchicago.edu/)

Cherenkov telescopes do not detect directly the $\gamma$-photon flux, but instead detect the Cherenkov light produced in the atmospheric air shower induced by the primary photon. Light emitted at the Cherenkov angle reaches the ground within a circle of $100 \mathrm{~m}$ to $150 \mathrm{~m}$ radius depending on the height above sea level of the detection system. An optical telescope pointing to the source and located within the illuminated footprint of the shower can detect the air shower against the background light of the night sky, provided the camera is sufficiently fast to integrate the short Cherenkov flash of the order of few nsec. Then, the Cherenkov technique takes advantage of the shower development information in the image of the telescope camera. It is therefore possible to take a sort of "snapshot" of the air showers resolved in space (and time). This information can then be used to distinguish the origin of the air shower (hadronic or $\gamma$-ray) using the different spatial development of $\gamma$ - and hadron-induced air showers. The parameterization of such images is called "Imaging Technique", which dramatically improves the $\gamma /$ hadron separation power and makes IACTs the most successful instrument for cosmic very high energy $\gamma$-ray observations.

The international communities working in the $\mathrm{TeV}$ astronomy both in Europe and USA are now involved in the study of huge arrays of Cherenkov telescopes such as the Cherenkov Telescope Array (CTA) [2] and the Advanced Gamma-ray Imaging System (AGIS) [3] projects. The proposed facilities of the next generation will consist of extended arrays of Cherenkov telescopes, aiming to (a) increase sensitivity by another order of magnitude for deep observations, (b) boost significantly the detection area and hence the detection rates, particularly important for transient phenomena and at the highest energies, (c) increase the angular resolution and hence the ability to resolve the morphology of extended sources, (d) provide wide and uniform energy coverage from some $10 \mathrm{GeV}$ to beyond $100 \mathrm{TeV}$ in the energy of the photons, and (e) enhance the all sky survey capability, the monitoring capability and the flexibility of operation. These new, complex and very large facilities need, among other things, a substantial technological development of many telescope subsystems. From the installation, operational and maintenance of each single telescope to the efficient coordination of the complete array in a structure organized as an observatory, from the science data center to the huge power supply.

The AGIS collaboration is intensively studying an array with a 36-telescope layout exploiting a more innovative Schwarschild-Couder S-C optical design [4]. The S-C telescope takes advantage of a double reflection configuration with strong aspherical surfaces. It shows a wide aplanatic field of view with almost constant angular resolution. In particular the secondary mirror of such instruments seems quite challenging from the manufacturing point of view because it has a pronounced concave surface, with sags of 30-50 $\mathrm{mm}$ on a single $1 \mathrm{~m}^{2}$ mirror panel.

Instead, CTA will implement a large array with a modular design composed of three different telescope types, namely the Small Size Telescopes (SST), the Medium Size Telescopes (MST) and Large Size Telescopes (LST), of 4-7m, 12m, and $24 \mathrm{~m}$ diameter respectively. The core part of the array is composed by some tens of MSTs whose sensitivity will be extended at the lower energies by a few LSTs. Meanwhile, the sensitivity curve at very high energies, above some TeV, will be boosted by an extended array of nearly hundred SSTs covering an effective area of some kilometers squared. In the left panel of Fig. 2 it is shown an artistic view of CTA. The final layout of these arrays is still under investigation through detailed Monte Carlo simulations, two different layouts are shown in Fig. 2 (central and right panels). 
CTA is ranked in ASPERA and ASTRONET and listed in the emerging proposals of ESFRI 2006. Moreover, in December 2009 a large Consortium encompassing 129 institutes from 22 countries submitted a proposal to the European Commission within the 7th Framework Program (FP7) for the Preparatory Phase for the Cherenkov Telescope Array (CTA-PP, FP7-INFRASTRUCTURES-2010-1). A letter sent in late March 2010 informed the consortium that this proposal has been favorably evaluated and funded.
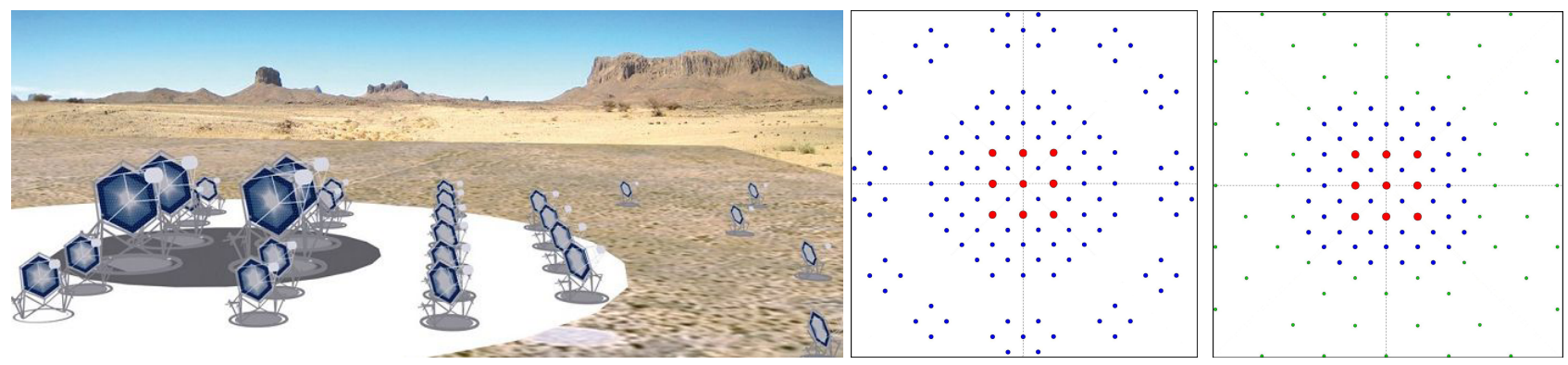

Fig. 2. (left) Artistic view of a possible CTA configuration, with three different telescopes types covering the overlapping energy ranges, and area coverage which increases with increasing gamma-ray energy. Two possible geometries of arrays, the colors represent different telescopes diameters: (center) array layout without small telescopes. The blue clustered points in the outer region provide the detection of the higher energies. (right) array layout with three classes of telescopes.

The Medium Size Telescope will probably adopt the classical single reflection Davies-Cotton optical design [5]. In the Davies-Cotton layout, generated originally for solar concentrators, all reflector facets have a spherical profile with the same focal length $f$, identical to the focal length of the telescope as a whole. The mirror facets are arranged on a dish having a spherical profile of radius $f$. Each MST will have a reflective area of more than $100 \mathrm{~m}^{2}$, composed of nearly 100 identical mirror panels. About 30 telescopes will be constructed for a total mirror area around $3000 \mathrm{~m}^{2}$. It is therefore very clear the importance of a proper technology for the mirrors production.

This paper is inserted in the framework presented above and focused on the aspects related to the mirrors development for CTA. In particular we report the status of the art of a novel technology under development in Italy at the Astronomical Observatory of Brera. This technology is called "cold glass slumping" and foresees the manufacturing of glass mirror panels with a sandwich-like structure.

The following sections 2 and 3 report a description of typical requirements of mirrors for Cherenkov telescopes and the technologies till now adopt for their production. Sections 4 and 5 respectively describe the preliminary design performed through Finite Element Analyses for a mirror panel of the MST class of telescopes of CTA, and the technology we are investigating together with some results obtained on prototypes realized so far. Section 6 reports some conclusions and future steps of our work.

\section{MIRROR SPECIFICATIONS}

Despite the final array configuration has not yet been chosen, it is required as order of magnitude, to have about $10000 \mathrm{~m}^{2}$ of total reflecting area with single telescope reflectors reaching up to $400 \mathrm{~m}^{2}$. For Cherenkov telescopes high collecting area is needed, achievable by the use of a large diameter primary, made of a mosaic of many (possibly lightweight) reflecting facets, like the ones needed for future next generation ground based optical telescopes such as the $42 \mathrm{~m}$ E-ELT telescope studied by ESO.

While in both cases a high volume production has to be achieved, for making the reflecting panels for Cherenkov telescopes the angular resolution is not an issue: each panel should present a PSF of the order of about few arcmin, compared to the 0.1 arcsec value requested for each E-ELT panel. The PSF should be anyway smaller than the pixel size over the entire field of view. 
Another peculiarity of Cherenkov telescopes is that mirrors are continuously exposed to the environment because the absence of a protecting dome. For such and other reasons the development of mirrors for large Cherenkov telescopes is non trivial. The technological goal is to manufacture mirrors with a number of challenging characteristics such as lowcost, light-weight, robustness, with an adequate reflectance and focusing qualities and requiring a limited maintenance. For example, they should maintain the cost below $2000 € / \mathrm{m}^{2}$ and weight below $25 \mathrm{~kg} / \mathrm{m}^{2}$; for comparison, the same target parameters for E-ELT are around $300 \mathrm{k} € / \mathrm{m}^{2}$ and $70 \mathrm{~kg} / \mathrm{m}^{2}$ respectively.

Main requirements are listed in [6][7] and here summarized:

Mirror tile. The geometry currently favored for the facets of the CTA telescopes is hexagonal in shape, with a size of $1.2 \mathrm{~m}$ face-to-face, well beyond the presently common size of $0.3-1 \mathrm{~m}^{2}$.

Weight. The weight limit depends on the characteristics of the telescope like the mirror alignment system and constraints due to the necessary handling/mounting of the mirrors. A total weight of less than $25 \mathrm{~kg} / \mathrm{m}^{2}$ would be desirable.

Rigidity. High rigidity of the mirror panels is a fundamental requirement. The mirror deformation under gravity must be small enough to maintain the specifications for the PSF and the alignment. This parameter has been evaluated in the following analyses through the slope deviation from the nominal profile of the mirror surface. Slope errors should remain below $0.5 \mathrm{mrad}$.

Temperature range. IACTs are normally placed at altitudes of 1000-3000 $\mathrm{m}$ a.s.l. where the daily temperature changes by several tens of degrees and rapid temperature drops are quite frequent. Mirrors should resist to temperature changes from $-15^{\circ} \mathrm{C}$ to $+60^{\circ} \mathrm{C}$ and the optical properties (i.e. the focal length and PSF) should not change significantly in the range $0^{\circ} \mathrm{C}$ to $+30^{\circ} \mathrm{C}$.

Point Spread Function. Intrinsic aberrations in the Cherenkov light emitted by atmospheric showers limit the angular resolution to values of around 30 arcsec. However, the requirement on the focusing quality (i.e. the spot size) of the mirrors is driven by the pixel size of the camera and the final design of the telescope reflector. Using the standard Cherenkov Imaging Technique, there is no real need to produce mirror facets with a PSF well below the half of the camera pixel size, which is ordinarily not smaller than few mrad.

Reflectance. The reflectance shall exceed $80 \%$ for all wavelengths in the range from $300 \mathrm{~nm}$ to $600 \mathrm{~nm}$, ideally close to (or even above) $90 \%$. The Cherenkov light intensity peaks between $300 \mathrm{~nm}$ and $450 \mathrm{~nm}$, therefore the reflectance of the coating should be optimized for this range.

Durability. CTA will be operated for at least 10 years. Therefore, the mirror facets should maintain their performance for that duration. The reflectance should not degrade by more than a few percent under the prevailing environmental conditions.

\section{TECHNOLOGIES}

Different technologies have been adopted so far for the production of the Cherenkov segmented mirrors. The different technologies can be divided into two main groups:

aluminized ground-glass mirrors They are manufactured with standard technique starting from raw blanks. Groundglass mirror solution has been often preferred (e.g. HEGRA, CAT, HESS and VERITAS) primarily because of its technical maturity, but at cost of a quite long time of production. Moreover, the ground-glass mirrors are quite heavy translating into increasing cost and complexity for the telescope mechanical structure;

composite sandwich structure mirrors In sandwich construction, membranes (such as sheets of steel, aluminum, glass, or plastic) are bonded to both sides of a core material. This type of construction is widely utilized in products ranging from doors and tables to aircrafts, boats and satellites and is characterized by high strength-to-weight. Also the MAGIC mirrors are composite structure:

- composite sandwich structure mirrors manufactured via direct machining of each individual piece [8][9]: in this case consisting of an aluminum face sheet roughly curved in autoclave to spherical shape and glued to an aluminum honeycomb inside a thin aluminum box making up a so called "raw blank". Each individual raw 
blank is subsequently polished by a diamond milling process. For the MAGIC II telescope 136 one squared meter mirrors have been manufactured in this way;

- composite sandwich structure mirrors manufactured via replication process from a mould [10][11]: the mirror elements have a sandwich like structure where the reflecting and backing membranes are bonded to both sides of an aluminum honeycomb core. The membranes are thin sheets of glass, aluminized for the reflecting one. For the MAGIC II telescope 104 one squared meter mirrors have been manufactured in this way.

The cold glass slumping technology we are proposing can be inserted in the class of composite sandwich structure mirrors manufactured via replication process. This is a new method derived from a similar technique proposed for the manufacturing of X-ray optics of the former XEUS mission [12], that fits the requirements of Cherenkov telescopes. It has been developed by the Media Lario Technologies company in collaboration with INAF-OAB. Media Lario Technology has been also in charge of the mirror panels production and quality control for MAGIC II.
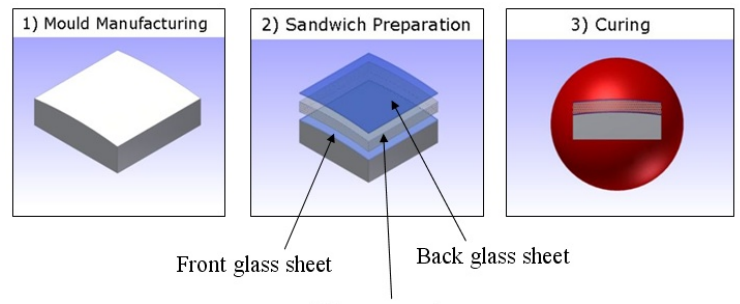

Al honeycomb core
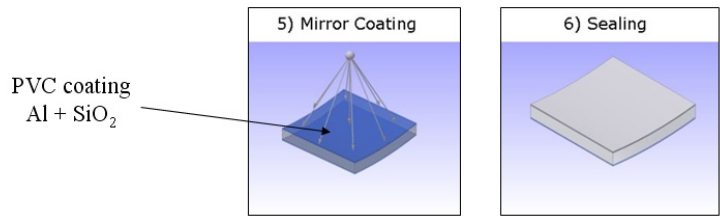

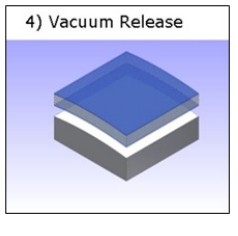

Fig. 3: Flow chart of the cold glass slumping technology. Credits: Media Lario Technologies.

The fabrication process of the cold glass slumping is hereafter described [11] and the main steps are shown in Fig. 3 :

- a master has to be worked with the same optical precision needed for the mirrors, because every defect on its surface will be reproduced on the glass. Diamond milling is used in order to achieve the best shape accuracy possible without the need for further polishing. The master has been produced by the LT-Ultra company (Germany). The master has a spherical convex curvature of the surface;

- the shape of the master is replicated by the reflecting glass sheet taking advantage that for large curvature radii the glass sheet can be elastically deformed and pressed against the master using vacuum suction. A backing glass sheet is assembled with an interposed aluminum honeycomb core element giving the proper rigidity. In principle from a single master one could think that only one radius of curvature for the mirror could be obtained. Nevertheless, by a careful control of the spring-back effect during the gluing process, one can obtain several mirrors with curvature different than the master's one;

- the connection of the parts is achieved through epoxy resin structural adhesive bonding with curing under elevated temperature while maintaining the vacuum suction;

- the glass sheets adopted are floating glass available on the market with a very good roughness and do not require any polishing step. The process ensures on the reflecting glass sheet the required shape accuracy after separation from the master and the preservation of the starting surface roughness of the glass sheet;

- the reflecting coating is deposited after the manufacturing of the sandwich structure by means of physical vapor deposition in a dedicated high vacuum chamber. The reflecting glass sheet is coated in order to provide a high reflectivity at wavelengths in the range from $300 \mathrm{~nm}$ to $600 \mathrm{~nm}$. Aluminum coating provides the best reflectivity at these wavelengths, especially in the range of short wavelengths (300 nm to $450 \mathrm{~nm}$ ) that contains most intensity of the Cherenkov light. To avoid oxidation of the aluminum layer, a protective coating of Quartz is also applied; 
- sealing of the sandwich structure borders is assured by a silicon based sealant. The edges of the sandwich have an external plastic PVC rim. This solution assures higher rigidity and mechanical protection of the mirror corners.

This method has been successfully adopted for the production of half of the reflecting surface of the MAGIC II telescope, the world largest optical reflector [10]. However, it requires some further development in order to fully satisfy the requirement of CTA. Taking advantage of the experience done with MAGIC II, we have recently started a development roadmap aimed to:

- improve the replication accuracy in terms of radius of curvature and shape's deviation from the mould;

- improve the repeatability of the optical quality exhibited by each panel;

- lower the final cost of the panel.

The way we are pursuing to reach these goals can be summarized in the following main points:

- use of thinner glass sheets translate in a more flexibility of the front skin. This permits an ease bend of the glass and hence a more detailed copy of the mould, in particular for the medium spatial frequencies band;

- replacing the honeycomb core with a stiffer structure. This can strongly reduce the spring-back effect to which the panel is subjected, it is especially true if the core part of the panel is pre-processed to accommodate the curvature of the mould. In this respect we are testing different types of glass foam materials to be used instead of the Aluminum honeycomb. Glass foam has a high stiffness and a better CTE match with the front/back glass skins;

- use of cheaper materials, in particular concerning the core part of the panel and the epoxy glue used to assemble together the sandwich;

- reduce the production's steps where possible, especially if they are critical and/or manpower consuming such as the sealing of the borders of the panels.

\section{MIRROR PRELIMINARY DESIGN}

In parallel to practical activities described in other sections, we have performed some FEA to design a proper mechanical structure for a mirror panel for the MST. We have investigated its behavior with different loads cases, in particular for operative and survival conditions.

Operative condition is defined when the loads acting on the panel are: gravity (any orientation) and wind pressure at $50 \mathrm{~km} / \mathrm{h}$. In such loads condition, the stiffness of the panel should be high enough so that the mechanical deformations are kept as low as possible and do not affect the optical properties of the mirror.

Survival condition is defined when the loads acting on the panel are: gravity (any orientation), wind pressure at $180 \mathrm{~km} / \mathrm{h}$ and snow weight. In such loads condition, the stiffness of the panel should be adequate to avoid breaks.

The analysis have been performed with the ANSYS software. It has been modeled a regular hexagon with dimension of $1.2 \mathrm{~m}$ face-to-face; the total area is about $1.25 \mathrm{~m}^{2}$. The panel has one concave side corresponding to the optical surface, with a radius of curvature of $32000 \mathrm{~mm}$; the other side is flat. The panel is supported by three fixed points located at $2 / 3$ of the inner radius, along the apothem. Fig. 4 shows the model generated.

About hundred configurations have been investigated varying:

- glass sheet material: BOROFLOAT ${ }^{\circledR} 33$ from Schott and Microfloat ${ }^{\mathrm{TM}}$ from Pilkington;

- glass sheet thickness: 0.7-1.1-1.75-2 mm and 1.2-1.4-1.5-1.7 mm, respectively for BOROFLOAT® 33 and Microfloat ${ }^{\mathrm{TM}}$;

- core material: F-type and T4-type Foamglas ${ }^{\circledR}$ and Earthstone ${ }^{\circledR}$;

- core thickness: 30-40-50-60 mm.

An initial selection has been done using the following acceptance criteria: a) maximum weight $<31 \mathrm{~kg} / \mathrm{panel}\left(<25 \mathrm{~kg} / \mathrm{m}^{2}\right)$ and b) maximum slope $\mathrm{P}-\mathrm{V}$ error $<0.5 \mathrm{mrad}$. 

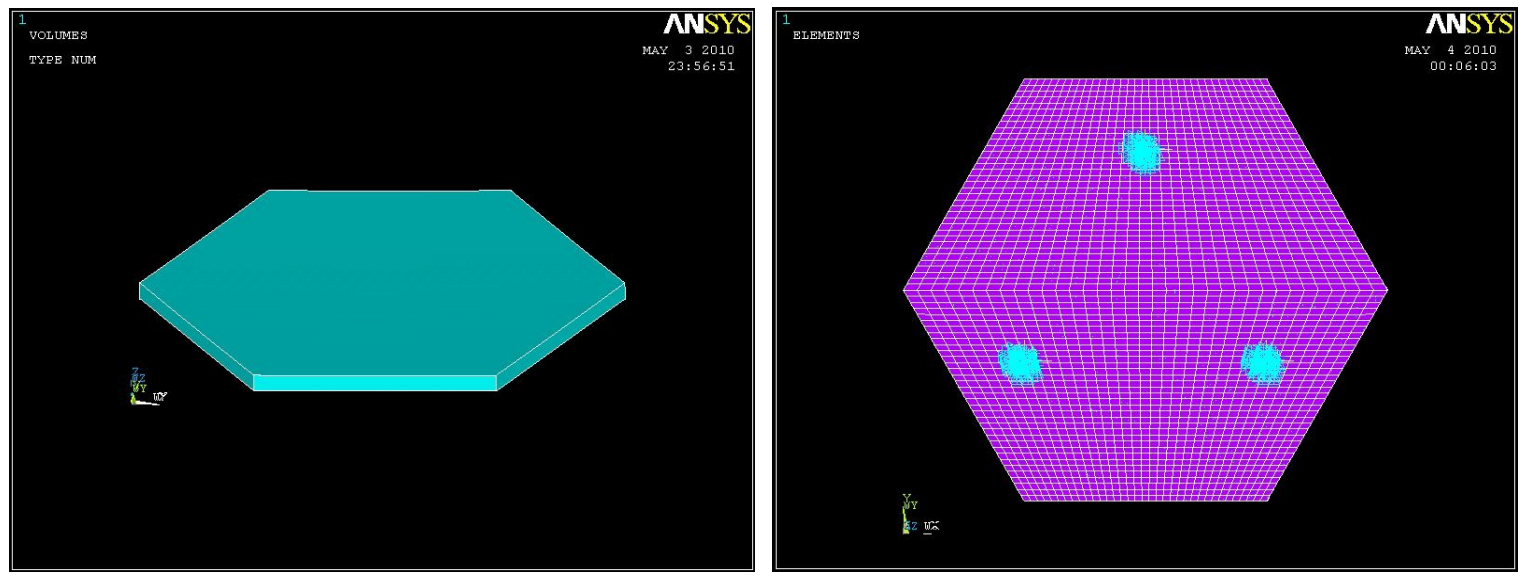

Fig. 4 (left) Model adopted with ANSYS to conduct the FEA. (right) Mesh and fixation used in the calculation.

\subsection{Loads determination}

During standard operation conditions, the panel is subjected to several forces. Each force generates deformations on the panel itself, and the panel should be stiff enough to counteract those effects. For design purpose we consider the worst situation in which the panel is placed parallel to the ground up-side and each loads act normally to the optical surface. Moreover, we have adopted more severe wind loads as reported in the following. The sum of the loads is passed to the three fixation points. Different loads are considered:

gravity load: as stated before the gravity force will act on the whole surface of the panel. The panel is supported by three circular pads of $40 \mathrm{~mm}$ in radius;

wind load: the wind acts as a kinetic pressure $\mathrm{P}_{\text {wind }}$ over the panel surface according to

$$
\mathrm{P}_{\text {wind }} \approx v^{2} / 2 \cdot \rho
$$

where $v$ is the wind speed and $\rho$ is the air density (varying with altitude). $\rho$ has been kept to its nominal value, i.e. the worst condition. We consider as upper limit for operative condition

$$
v_{\text {wind,op }}=65 \mathrm{~km} / \mathrm{h} \rightarrow \mathrm{P}_{\text {wind,op }} \sim 0.2 \mathrm{kPa}
$$

whereas, the upper limit in case of violent wind storm or hurricane is

$$
v_{\text {wind,surv }}=200 \mathrm{~km} / \mathrm{h} \rightarrow \mathrm{P}_{\text {wind,surv }} \sim 1.9 \mathrm{kPa}
$$

snow load: since the telescope is not protected by any dome, snow can deposits and accumulate on the reflector. We consider as upper limit a layer of snow of $30 \mathrm{~cm}$. Since, the density of the snow is about $30 \%$ of the density of water, over an area of $1 \mathrm{~m}^{2}$ are deposited about $90 \mathrm{~kg}$, i.e. $\mathrm{P}_{\text {snow }}=0.9 \mathrm{kPa}$.

\subsection{Selected configurations}

Since many configurations were compliant, we decided to add more acceptance criteria and to tighten the above ones as follow: a) maximum weight $<20 \mathrm{~kg} /$ panel $\left(<16 \mathrm{~kg} / \mathrm{m}^{2}\right)$ and b) maximum slope $\mathrm{P}-\mathrm{V}$ error $<0.3 \mathrm{mrad}$. The added criteria are: c) for overall cost reasons (in case of mirrors production) we discarded the BOROFLOAT ${ }^{\circledR} 3$ and d) from practical experiments the F-type Foamglas ${ }^{\circledR}$ seems to be the better core material.

In this way we can restrict the detailed study to the four configurations reported in Tab. 1.

The best trade-off configuration is the \#2, composed by glass sheets $1.2 \mathrm{~mm}$ thick and a core of $40 \mathrm{~mm}$ thick. This configuration provides very good values in terms of panel's weight, slope P-V and maximum stress developed on the core. Figure 5 shows the results from FEA performed on the configuration $\# 2$. 


\begin{tabular}{|c|c|c|c|c|c|c|c|}
\hline Conf. ID & $\begin{array}{c}\text { Glass } \\
\text { thickness } \\
{[\mathrm{mm}]}\end{array}$ & $\begin{array}{c}\text { Core } \\
\text { thickness } \\
\text { [mm] }\end{array}$ & $\begin{array}{c}\text { Areal density } \\
{\left[\mathrm{kg} / \mathrm{m}^{2}\right]}\end{array}$ & $\begin{array}{l}\text { Slope } \\
\text { P-V* } \\
\text { [mrad] }\end{array}$ & $\begin{array}{c}\boldsymbol{z} \text { displacement } \\
\mathrm{P}-\mathrm{V}^{*} \\
{[\mu \mathrm{m}]}\end{array}$ & $\begin{array}{c}\text { Total stress } \\
\text { into core }{ }^{* *} \\
\text { [MPa] }\end{array}$ & $\begin{array}{c}\text { Total stress } \\
\text { on glass** } \\
\text { [MPa] }\end{array}$ \\
\hline 2 & 1.2 & 40 & 11.89 & 0.092 & 37.9 & 1.1 & 26.5 \\
\hline 5 & 1.2 & 50 & 13.54 & 0.063 & 25.3 & 0.92 & 20.5 \\
\hline 7 & 1.7 & 30 & 12.74 & 0.134 & 53.2 & 1.21 & 31.8 \\
\hline 8 & 1.2 & 60 & 15.20 & 0.047 & 18.6 & 0.86 & 16.8 \\
\hline
\end{tabular}

Tab. 1: Configurations resulting after the application of the selection criteria.

*Slope and displacement P-V are referred to operative condition loads; **stresses are referred to survival condition loads.
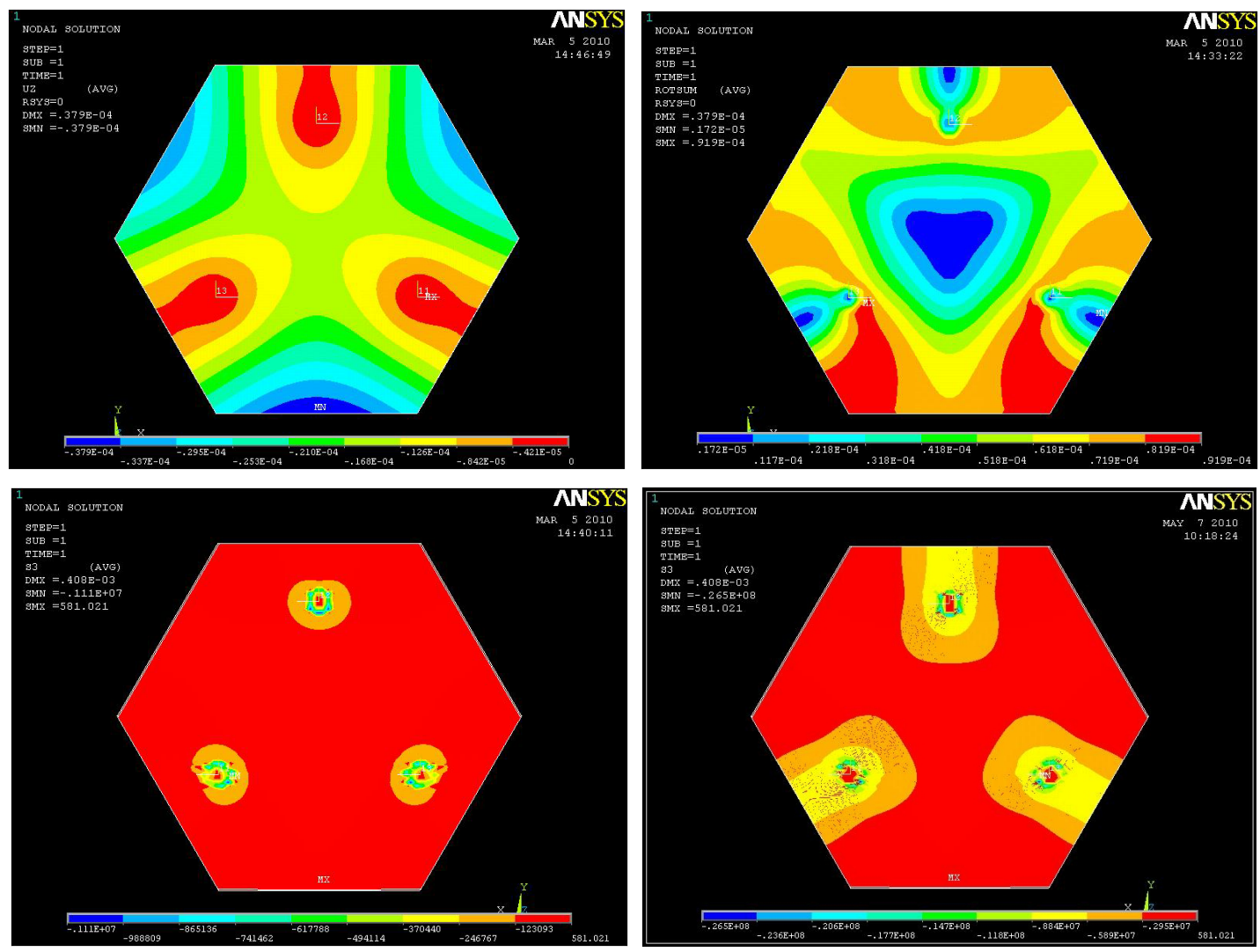

Fig. 5: Results from the FEA made on the configuration \#2. (top-left) Displacement on the optical surface, surface P-V; (top-right) Sum of the slopes on the optical surface, slope P-V; (bottom-left) $3^{\text {rd }}$ principal stress developed on the foam core, maximum stress. (bottom-right) $3^{\text {rd }}$ principal stress developed on the back glass sheet, maximum stress.

On the four selected configurations have been investigated also the behavior in extreme conditions. As described before, this condition is that where the panel must survive against strong atmospheric turbulence. The optical performances of the panel are not of interest, while the stress suffered by the various materials which compose the panel itself must not exceed their Yield strength limits.

Table 1 reports also the maximum values of the stresses ( $3^{\text {rd }}$ principal stress) developed on the foam and on the glass sheets. The glass sheets do not experience problems of survival since the maximum stress developed is well below the Yields strength of $100 \mathrm{MPa}$ (typical value for glass). Opposite situation occurs on the foam, where in some 
configurations it is approached the Yields strength of 1.6 MPa. Even if all the values obtained are below that limit, it is important to provide a good margin of safety (the value of 1.6 MPa already takes a factor two of safety).

Hence, the value of stress developed on the foam becomes the most important parameter to be evaluated. However, we should also notice that these conditions are very extreme and unusual and the site for CTA is not yet chosen, but this does not alter the nature of being even more violent.

\section{PRELIMINARY RESULTS FROM PROTOTYPES}

In the present section are reported a number of results obtained from mirror panel prototypes realized by the authors. The prototypes have been realized exclusively using equipments available in INAF-OAB's labs, in particular the mould comes from the mirrors production of MAGIC II. It has a squared tile shape of $1040 \mathrm{~mm}$ side, while the surface is a convex sphere of about $36300 \mathrm{~mm}$ radius of curvature. The measured figure $\mathrm{P}-\mathrm{V}$ is of about $21 \mu \mathrm{m}$ and the rms of $4.5 \mu \mathrm{m}$. Moreover, all the measurements (such as PSF spot size) and tests (such as thermal cycling) here reported have been performed using equipments installed in our labs, except when different stated.

\subsection{Glues}

The adhesive is an important structural component of these reflectors. It is used to join together the two sheets of glass with the core. It must ensure the bonding between the porous/irregular surface of the foam (or honeycomb or whatever) and the smooth surface of the glass.

From the practical point of view, the glue should be easy to handle and be prepared, this facilitates and speeds up the assembly of the panel. It should not introduce excessive stresses on the surface of foam to avoid to weaken the cellular structure of the surface of the foam.

From the optical point of view, it is preferable that the glue polymerizes at room temperature, it has the lowest possible shrinkage and does not introduce stress on the surface of the glass sheet so as not to deform its optical shape. It should also exhibit a limited aging upon ultraviolet radiation exposure (daily solar exposure).

Hence, both the technique adopted for gluing and the glue itself are very important.

EA Hysol 9309.3NA from Henkel Corporation Aerospace Group is commonly used in aeronautics and it is specifically designed to bond glass with Aluminum honeycomb. It has been used for the production of the 100 mirrors for MAGIC II; it shows the characteristics suitable for this application. In the following, the Hysol will be used as reference; it is the only glue used, till now, to assemble prototype mirrors with foam core.

Other adhesives have been looked for; in particular to deliver suitable performances for our application. One additional point taken into account on the survey was the cost impact. In total, three new adhesives have been investigated, namely: P-82F from Bacon Industries, Ocean EpoxyCrystal and Uretan NG from Cores. Tests were performed on bonding strength and aging to UV radiation.

To evaluate the bonding strength exercised by the glue between the sheet of glass and the core of the panel, we have performed a dedicated experiment. The experiment has been conducted in close collaboration with the Polytechnic of Milan by using a 858 Mini Bionix ${ }^{\circledR}$ II equipment. The specimens, albeit small, were representative of the sandwich structure of a real panel.

The sides of each specimen were glued with the same quantity of glue. The amount used ensures a bonding layer of about $0.3 \mathrm{~mm}$ thick. The glue was dripped in the center of the surface of the foam, verifying the amount with an accurate balance. Then, the glass plate was carefully lowered and was applied a little pressure with the fingers so that the glue spreads out circularly and evenly up to cover the entire bonding surface. Finally, the glue in excess (if any) was removed. The specimens were cured in an oven at about $50^{\circ} \mathrm{C}$ for about 2 days (see Fig. 6 left).

On both sides of the specimens were glued two Aluminum interfaces comprising the fastening system to the traction machine. The equipment was programmed to exert an axial traction force. Each specimen was anchored to the machine (see Fig. 6 center), a tensile force was applied and has been measured and recorded the elongation which occurred. Evaluating the tensile strength at the rupture can be assessed the overall glass-core-glass system and/or the glue only (respectively, if the core breaks or if the core separates from the glass plate). 

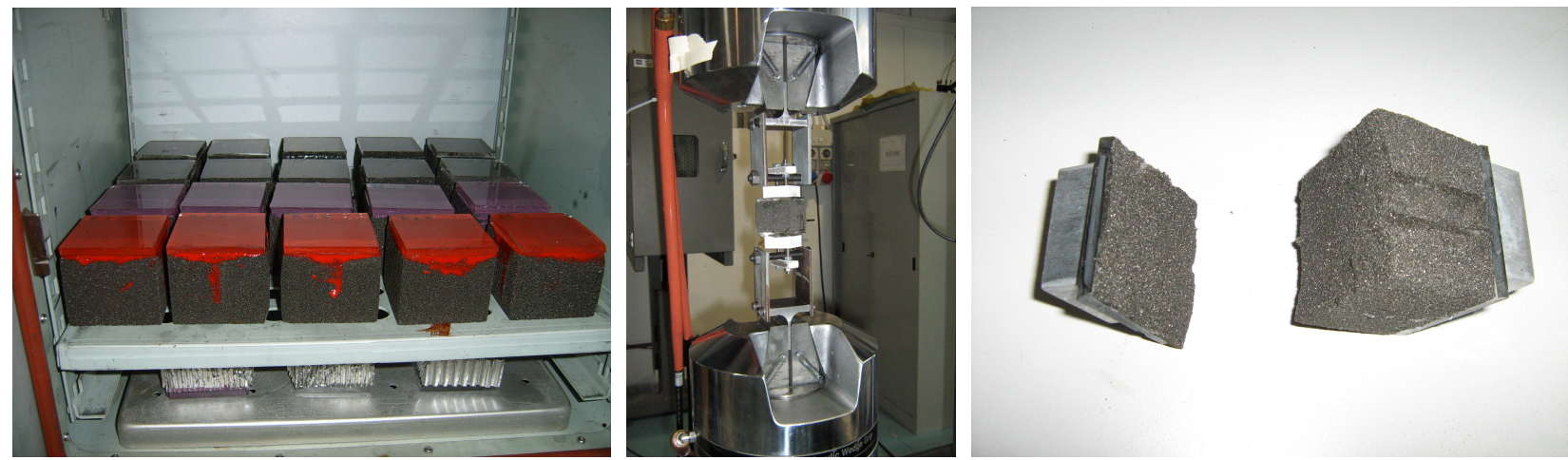

Fig. 6: (left) specimens during the curing of the glues (center) specimen mounted on the traction machine (right) specimen after the test.

\begin{tabular}{|l|c|c|c|c|}
\cline { 2 - 5 } \multicolumn{1}{c|}{} & \multicolumn{3}{c|}{ Epoxy Adhesives } \\
\hline \multicolumn{1}{c|}{ Properties } & Hysol & Bacon Industries & Cores \\
EA9309.3NA & P-82F & Ocean EpoxyCristal & Cores \\
Uretan NG
\end{tabular}

Tab. 2: Properties of the four epoxy glues investigated. Values in brackets are measured by the authors.

Summarizing, Tab. 2 lists the main characteristics of the glues, some of them come from datasheet and some other come from practical experience.

The Ocean EpoxyCrystal has mechanical performances comparable to those of the Hysol, less aging due to UV irradiation and an easier applicability. Moreover, it introduces some other interesting advantages (such as the low temperature needed for the curing) that makes it worthy of being further investigated. Last but not least, it allows a considerable cost saving.

\subsection{Making of a prototype}

The purpose of this activity is to optimize the technique of the cold glass slumping respect to the construction of mirrors with glass foam core [13]. The prototypes realized have two thin glass skins made of BOROFLOAT ${ }^{\circledR} 33$ and a core made of F-type Foamglas ${ }^{\circledR}$. The glass sheets are $1.1 \mathrm{~mm}$ thick, while the core is $40 \mathrm{~mm}$ thick. The core has been machined to allocate the curvature of the mould. Figure 7 illustrates the different phases of the manufacturing. 


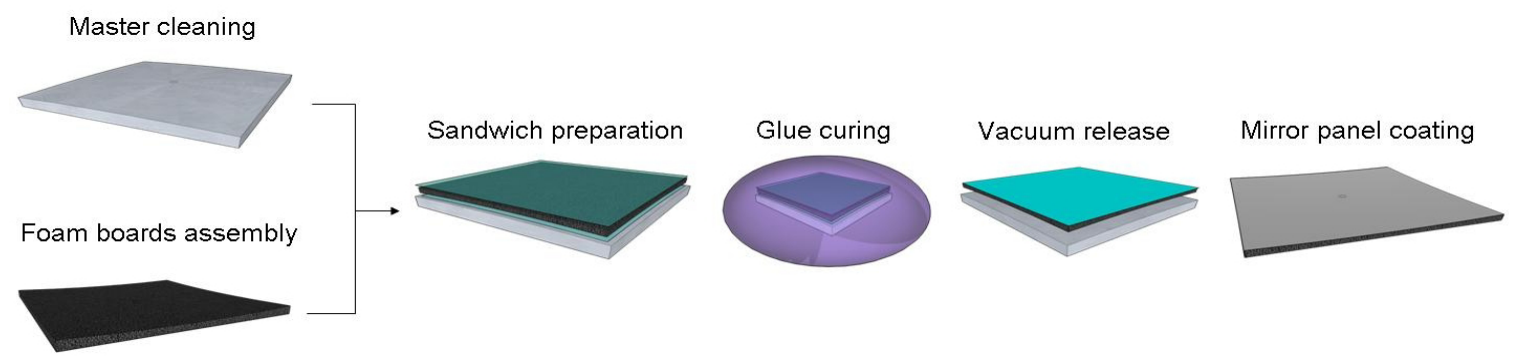

Fig. 7: Flow chart for the cold glass slumping technique using foam core.

The procedure for the realization of a prototype can be described as follow:

Phase 1: prepare the Foamglas ${ }^{\circledR}$ board and the core of the panel. Foam boards are flattened and assembled together on a glass sheet to form a solid body. This glass sheet will be the back plate of the panel (see Fig. 8 (top-left)).

Phase 2: machining of the core to match the curvature of the mould. This part has been done using an ad hoc equipment capable of milling the foam to the proper shape (see Fig. 8 (top-right)). One squared meter is milled in about a hour.

Phase 3: slumping of the front glass sheet and spread of the glue. It is done using a vacuum suction to make adhere the glass to the mould. The glue was spread using a proper tool (with knife edge) that permits to have a uniform thin layer of about $0.3 \mathrm{~mm}$ thickness (see Fig. 8 (bottom-left)).

Phase 4: the core is bonded to the front glass sheet and the overall system is cured. The polymerization is obtained heating up the mould up to about $80^{\circ} \mathrm{C}$ for few hours (see Fig. 8 (bottom-right)).

We have realized a number of small prototypes. The panels are squares of $300 \mathrm{~mm}$ by side and are assembled using the Hysol adhesive. Later on, it has been realized also a larger prototype of $600 \mathrm{~mm}$ by side.
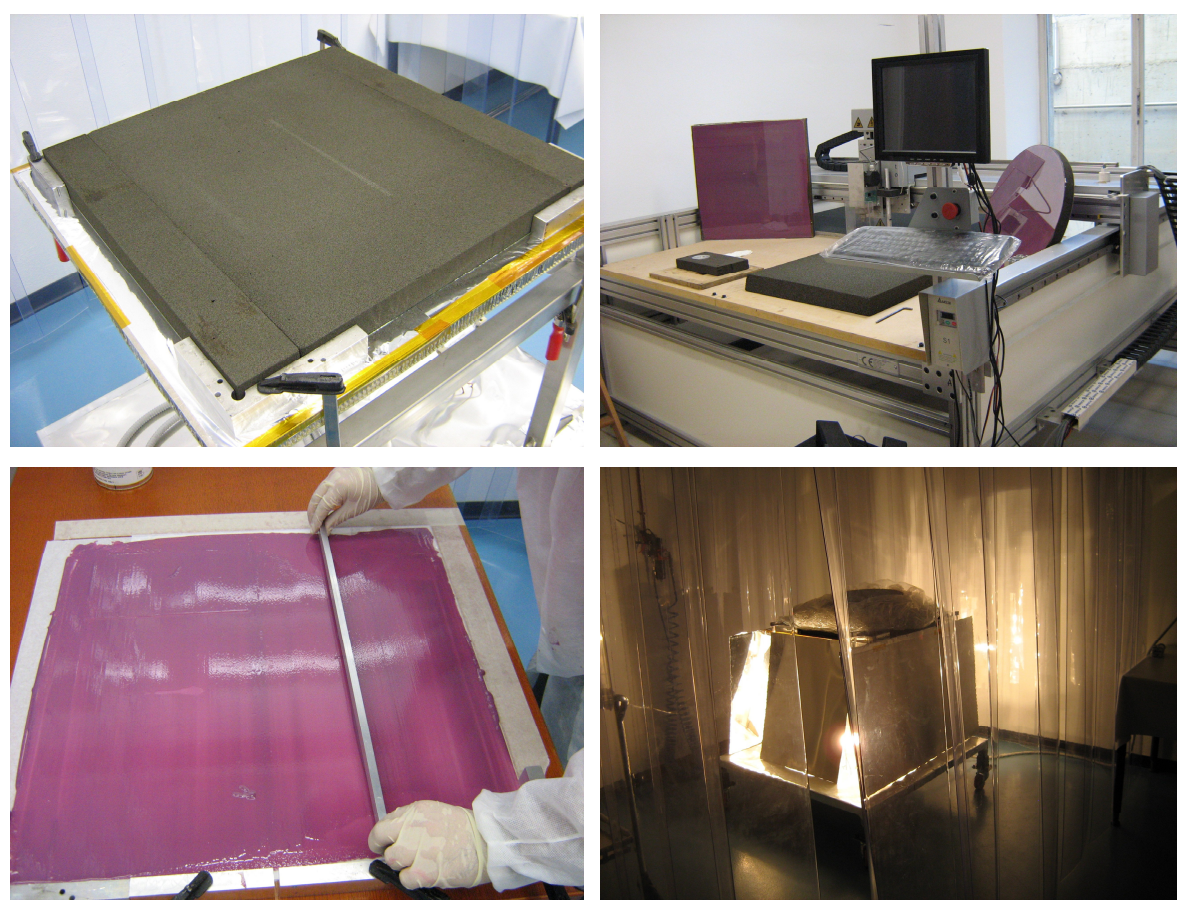

Fig. 8: Main phases of the realization of a prototype with F-type Foamglas ${ }^{\circledR}$ core. (top-left) Preparing the core, foam boards assembling. (top-right) Core machining. (bottom-left) Spreading out the Hysol glued. (bottom-right) Curing of the glue. 


\subsection{Qualitative inspection}

Figure 9 (top-left) shows an aluminized small panel prototype. As visible, there is a clear effect on the edges coming from the replication process. In fact, to protect the soft surface of the mould from the deterioration that could arise from the process itself, we use a thin foil of a breathable material. This foil prevents the complete contact of glass edges with the mould's surface and hence, the shape of the glass sheet deviates (locally) from that of the mould. In Fig. 9 (top-right) is visible, projected on a building wall, the image of the Sun generated by the panel. The spot is round and well defined, this means that the edges do not affect in a significant way the focusing quality of the mirror.

Figure 9 (bottom-left) shows the larger prototype till now realized: the $600 \times 600 \mathrm{~mm}^{2}$ panel after the vacuum release. The panel is not aluminized and the purple color comes from the Hysol glue. The panel has an areal density of about $12 \mathrm{~kg} / \mathrm{m}^{2}$, a value fully into specs for what required by the next generation of Cherenkov telescopes. Once again, in Fig. 9 (bottom-right) is visible the image of the Sun generated by the panel. The spot is round but less defined than the previous one, this means that the process needs to be refined going to larger dimensions.
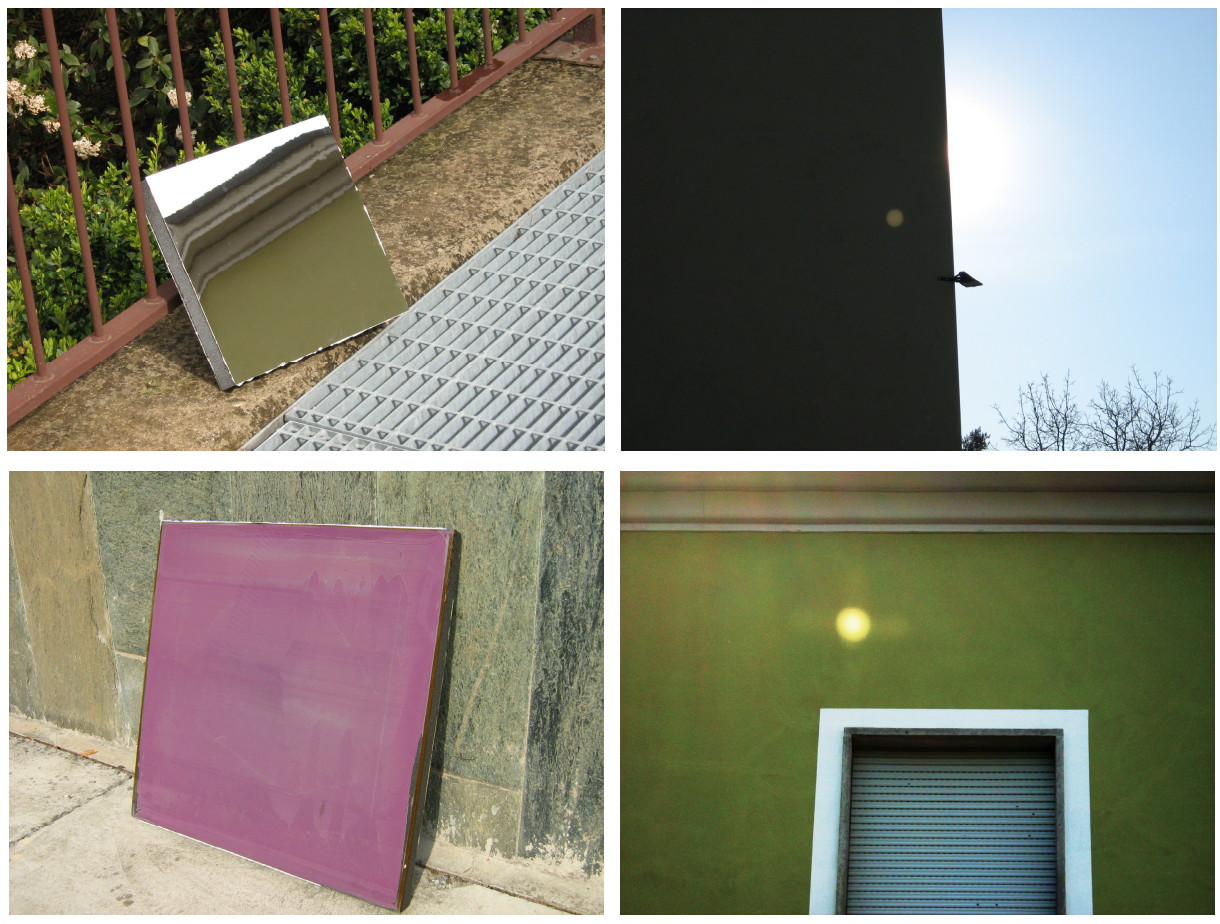

Fig. 9: Prototypes made with F-type Foamglas ${ }^{\circledR}$ core. (top-left) $300 \times 300 \mathrm{~mm}^{2}$ prototype, the surface is aluminized. (topright) Image of the Sun generated by the small prototype. (bottom-left) $600 \times 600 \mathrm{~mm}^{2}$ prototype, the surface is not aluminized. (bottom-right) Image of the Sun generated by the larger prototype.

\subsection{PSF spot size and curvature radius}

Due to the observation technique adopted by the Cherenkov telescopes, the FWHM is not the best parameter to evaluate such mirrors. Since the major part of the light should be contained into a single pixel, it is more convenient to define a stricter parameter: the radius containing the $90 \%$ of the focused energy, in the following indicated as $r_{90}$.

The measurements have been done using the long horizontal optical bench. The mirror is placed at a distance of about $36 \mathrm{~m}$ (the assumed radius of curvature of the mirror) and illuminated with a point light source. The point-like light source has been simulated by a laser diode with a microscope objective and a pin-hole in front. The distance between the mirror and the point source is equal to the nominal curvature radius (or twice the focal length) of the mirror itself, in such a way a point image is reflected again into a point image. A screen is placed close to the diode, at the same distance to 
the mirror. The distance from the mirror and the diode was adjusted searching for the best image position. The reflected spot was imaged with a 16 bit CCD camera and used for the PSF evaluation test.

In Fig. 10 (left) is shown a typical focal spot generated by a $300 \mathrm{~mm}$ side prototype. The value for the $\mathrm{r}_{90}$ is of about $0.25 \mathrm{mrad}$, well below the requirements. However, the best focus position has been measured at about $32000 \mathrm{~mm}$, a value quite less than what expected. This behavior was already noticed during the production of the mirrors for MAGIC II. In particular the mirrors show a radius typically shorter of about $800 \mathrm{~mm}$. In this case the variation exceeds this amount. Indeed, several identical panels have been replicated from the same position onto the mould's surface, and we are confident that the error is due to a local deviation of the mould shape from the nominal one. In fact, the replication of a larger area of the mould minimizes this effect.

Also for the $600 \mathrm{~mm}$ side panel we have measured the focal spot size as reported in Fig. 10 (right). As argued from the evaluation of the Sun's image, the $r_{90}$ is worse and not yet fully in specs (but still very close). The value is of about $0.6 \mathrm{mrad}$. Nevertheless, masking the mirror so to measure just its central part the value drops to about $0.35 \mathrm{mrad}$. As claimed before, the best focus position is (as expected) slightly less than the mould's radius, it is at about $35800 \mathrm{~mm}$.

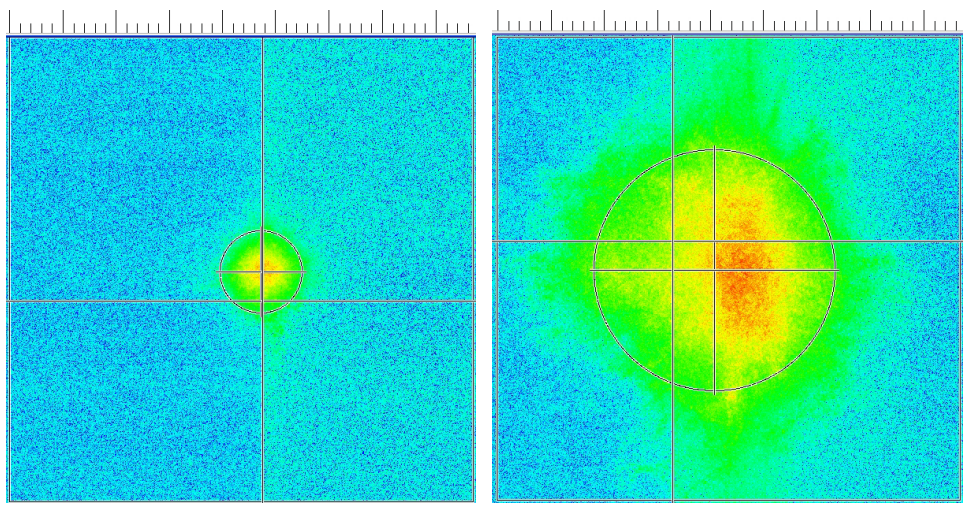

Fig. 10: PSFs spot size for prototypes made with F-type Foamglas ${ }^{\circledR}$ core. (left) $300 \times 300 \mathrm{~mm}^{2}$ prototype, best focus position at $32000 \mathrm{~mm}$. (right) $600 \times 600 \mathrm{~mm}^{2}$ prototype, best focus position at $35800 \mathrm{~mm}$.

\subsection{PSF after thermal cycling}

In addition, panels have been tested also after undergoing thermal cycling. The adopted cycles were spanning from $-20^{\circ} \mathrm{C}$ up to $+60^{\circ} \mathrm{C}$, each thermal cycle lasted several hours (see Fig. 11 left). The PSF spot size of a few mirrors, with dimensions of $300 \times 300 \mathrm{~mm}^{2}$, has been checked after the thermal cycle. This has been repeated for a number of times; the results have been compared to check degradations of the focusing quality of the mirrors (see Fig. 11 right). Till now the mirrors tested did not have shown any appreciable degradation of the $r_{90}$. However, this result must be carefully considered due to the very small dimensions of the prototypes tested. Nevertheless, it is an encouraging result that must be repeated on full scale panels since these kinds of mirrors have to be intensively tested for survival.
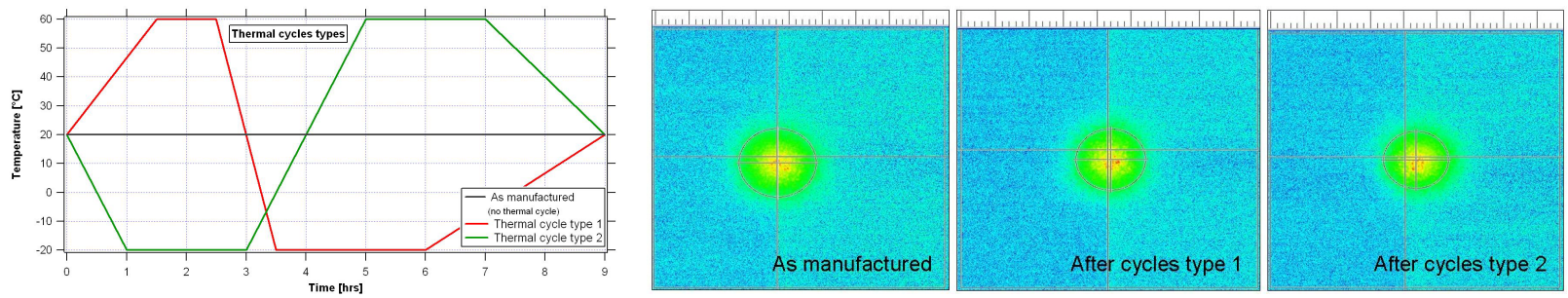

Fig. 11: (left) Thermal cycles used to test the prototypes (right) PSFs spot size for prototypes made with F-type Foamglas ${ }^{\circledR}$ core. 


\section{CONCLUSIONS}

In this paper is described the innovative technique ideated and developed by INAF-OAB expressly for mirror panels for Imaging Atmospheric Cherenkov Telescopes. These panels have a composite sandwich-like structure composed by two skins of thin borosilicate glass sheets and a reinforcing core. Despite they require a limited optical quality (few arcminutes are sufficient), the main features of this kind of mirrors should be robustness to strong environmental and atmospheric conditions, reduced weight and limited cost.

The authors have contributed to study and develop the "Cold Glass Slumping" process. The process is based on the replica concept, where a number of identical objects can be produced in a fast and cheap manner coping the shape of the mould. In this process we elastically deform a thin $\left(1-2 \mathrm{~mm}\right.$ ) and large (about $1 \mathrm{~m}^{2}$ ) borosilicate glass sheet onto the surface of an accurate mould by means of a vacuum suction. The mould is made in Aluminum, it has the negative profile of the desired mirror and it is figured through a diamond milling fly cutting technique. The goal of the process is to copy within the requested accuracy its shape and to retain it when the suction is stopped.

Using this technique have been already produced with success about half of the mirror panels of the MAGIC II Cherenkov telescope. Nowadays, a further investigation is part of a larger effort dedicated to the CTA FP7 Preparatory Phase.

At this regard, the paper describes and reports the latest advancements gained. The authors are conducting a step further in the development of the technology to make it fully compliant to the requirements of CTA. In particular concerning the cost target we are investigating innovative and cheapest materials such as glass foam and glues; this materials come from different markets (than optics manufacturing) and need to be characterized.

The authors have realized and tested with success a number of $300 \times 300 \mathrm{~mm}^{2}$ mirror prototypes. These panels have focusing performances typically below $1 \mathrm{mrad}$ as requested for Cherenkov telescopes. They survived to a number of rapid thermal cycles from $-20^{\circ} \mathrm{C}$ to $+60^{\circ} \mathrm{C}$ with no appreciable deformations of the focal spot. Also, it has been realized and tested a larger size prototype $\left(600 \times 600 \mathrm{~mm}^{2}\right)$ showing a focusing quality close to $1 \mathrm{mrad}$. All these results are very promising in view of using glass foam as core material for the reflecting panels. Nevertheless, these new kind of materials have to be intensively tested.

In the next months will be realized a full scale mirror panel compliant with the CTA requirements and adopting the new materials. A detailed schedule of tests is going to be defined to check the focusing performances, survival and operational conditions, aging and reflectivity.

\section{ACKNOWLEDGMENT}

This work has been done in the framework of the Mirror Working Group of the CTA and AGIS collaborations.

I would acknowledge INAF, in particular Bruno Sacco and Stefano Vercellone for many useful discussions. Also thanks to Astronomical Observatory of Brera for supporting with internal funds this work, in particular Giovanni Pareschi to encourage me in pursuing these developments.

I would like to thank Marco Riva, Gaetano Marino and Politecnico di Milano in giving us help and support for the mechanical characterization of glass foams and glues.

\section{REFERENCES}

[1] Weekes, T. C., et al., "Observation of $\mathrm{TeV}$ gamma rays from the Crab nebula using the atmospheric Cerenkov imaging technique", The Astrophysical Journal, 342:379\{395\}, July 1989.

[2] Hermann, G., "The ground-based gamma-ray observatory CTA", Astronomische Nachrichten, 2007, vol.328, issue7

[3] "The status and future of ground-based TeV gamma-ray astronomy", AGIS White Paper

[4] Vassiliev, V. V., Fegan, S. J., Brousseau, P. F., "Wide field aplanatic two-mirror telescopes for ground-based gamma-ray astronomy", Astropart. Phys, 2007, vol. 28, issue 1, p. 10-27

[5] Davies, J. M., Cotton, E. S., "Design of the Quartermaster Solar Furnace”, Solar Energy Sci. Eng. 1 (1957) 16-22. 
[6] Foerster, A., et al., "Mirror development for CTA", Proc. SPIE 7437, 743712 (2009)

[7] Doro, M. et al., "Mirror Facet Technologies for the Telescopes of the CTA Observatory", Proceedings of the $31^{\text {st }}$ ICRC, Łódź 2009

[8] Bigongiari, C., et al., "The MAGIC telescope reflecting surface”. Nuclear Instruments and Methods in Physics Research A, 518:193-194, February 2004.

[9] Doro, M., et al., "There Reflective surface of the MAGIC telescope". Nuclear Instruments and Methods in Physics Research A, 595:200-203, September2008.

[10] Pareschi, G., et al., "Glass mirrors by cold slumping to cover $100 \mathrm{~m}^{2}$ of the MAGIC II Cerenkov telescope reflecting surface”. Proc. SPIE 7018, (2008).

[11] Vernani, D., et al., "Development of cold-slumping glass mirrors for imaging Cerenkov telescopes". Proc. SPIE 7018 (2008).

[12] Ghigo, M., et al., "The manufacturing of the XEUS x-ray glass segmented mirrors: status of the investigation and last results". Proc. SPIE 5168 (2004).

[13] Canestrari, R., et al., "Techniques for the manufacturing of stiff and lightweight optical mirror panels based on slumping of glass sheets: concepts and results”. Proc. SPIE 7437 (2009). 